\title{
Selective Heating of Transition Metal Usings Hydrogen Plasma and Its Application to Formation of Nickel Silicide Electrodes for Silicon Ultralarge-Scale Integration Devices
}

\author{
Tetsuji Arai ${ }^{*}$, Hiroki Nakaie1, Kazuki Kamimura1, Hiroyuki Nakamura1, \\ Satoshi Ariizumi', Satoki Ashizawa1, Keisuke Arimoto', Junji Yamanaka1, \\ Tetsuya Sato1, Kiyokazu Nakagawa1, Toshiyuki Takamatsu ${ }^{2}$ \\ ${ }^{1}$ Interdisciplinary Graduate School of Medicine and Engineering, University of Yamanashi, Kofu, Japan \\ ${ }^{2}$ SST Inc., Yachiyo, Japan \\ Email: "g15df001@yamanashi.ac.jp
}

Received 20 October 2015; accepted 5 January 2016; published 11 January 2016

\begin{abstract}
We developed an apparatus for producing high-density hydrogen plasma. The atomic hydrogen density was $3.1 \times 10^{21} \mathrm{~m}^{-3}$ at a pressure of $30 \mathrm{~Pa}$, a microwave power of $1000 \mathrm{~W}$, and a hydrogen gas flow rate of $10 \mathrm{sccm}$. We confirmed that the temperatures of transition-metal films increased to above $800^{\circ} \mathrm{C}$ within $5 \mathrm{~s}$ when they were exposed to hydrogen plasma formed using the apparatus. We applied this phenomenon to the selective heat treatment of nickel films deposited on silicon wafers and formed nickel silicide electrodes. We found that this heat phenomenon automatically stopped after the nickel slicidation reaction finished. To utilize this method, we can perform the nickel silicidation process without heating the other areas such as channel regions and improve the reliability of silicon ultralarge-scale integration devices.
\end{abstract}

\section{Keywords}

Selective Heating, Nickel Silicide Electrode, Hydrogen Plasma, Microwave Plasma

\section{Introduction}

Heat treatment is important for forming electrodes for silicon ultralarge-scale integration (Si-ULSI) devices [1]. The process temperature sometimes reaches approximately $800^{\circ} \mathrm{C}$. Therefore, we must consider that heat treatment causes the degradation of Si-ULSI reliability. To reduce the effects of heat treatment, we use laser annealing [2]-[4] to achieve selective heating. However, laser annealing requires a complex and expensive apparatus. To overcome these problems, we have proposed a simple heating method, in which transition metals can be heated selectively by exposing them to hydrogen plasma.

"Corresponding author. 
Mozetic and coworkers [5]-[10] reported that the temperature of nickel was increased by the irradiation of hydrogen atoms and reached approximately $300^{\circ} \mathrm{C}$, and from this phenomenon they estimated the density of hydrogen atoms in hydrogen plasma. This phenomenon is attributed to the release of binding energy by the recombination of hydrogen atoms into molecules on nickel surfaces, and as a result, the nickel is heated. The atomic hydrogen density $\left(\mathrm{N}_{\mathrm{o}}\right)$ is calculated from the first derivative of the nickel film temperature.

$$
N_{0}=\frac{8 M C_{p}}{v W_{D} S \gamma} \frac{d T}{d t}
$$

Here, $M$ is the mass of the nickel film, $C_{p}$ is its heat capacity, $v$ is the mean velocity of hydrogen atoms at room temperature, $\gamma$ is the recombination coefficient, $\mathrm{S}$ is the area of the nickel film, $W_{D}$ is the dissociation energy of a hydrogen molecule (4.5 eV), and $d T / d t$ is the temperature derivative with respect to the elapsed time.

We have developed a heating apparatus to form high-density hydrogen plasma, in which transition metals such as nickel and tungsten can be heated to approximately $1000^{\circ} \mathrm{C}$. In this paper, we report the maximum temperatures of transition-metal species obtained using the apparatus. Nickel is one of the most important transition metals because the source-drain electrodes of field-effect transistors in Si-ULSI are composed of nickel-silicon compounds; therefore, we also study the application of the heating method to nickel silicide formation for Si-ULSI.

\section{Experimental Methods}

We developed an experimental apparatus with a self-tuning microwave plasma system [11] (SST Inc. JX0-1500S1), as shown in Figure 1. This apparatus has four components: a reaction chamber, a $2.45 \mathrm{GHz}$ microwave generator, a gas flow control unit, and a rotary pump evacuation system. The reaction chamber has four parts: (a) an outer cylindrical chamber made of a dielectric material, (b) a microwave coupling section mounted on the surface of the outer cylindrical chamber, (c) an inner cylindrical chamber made of quartz (diameter: $131 \mathrm{~mm}$, length: 206 $\mathrm{mm}$ ), and (d) an aluminum chamber that surrounds the outer cylindrical chamber except for in the microwave coupling section. The output power of the microwave was between 50 and $1500 \mathrm{~W}$.

In all the experiments, the input microwave power was $1000 \mathrm{~W}$, the hydrogen gas flow rate was $10 \mathrm{sccm}$, and the pressure was $30 \mathrm{~Pa}$. The samples were set on a sample stage made of quartz and exposed to hydrogen microwave plasma. We evaluated the sample temperature using a radiation thermometer with a measuring wavelength ranging from 0.8 to $1.6 \mu \mathrm{m}$ (Japan Sensor Corporation FTK9-P300R-30L22) through a quartz window.

To observe the heat phenomenon of various materials during hydrogen plasma exposure, we prepared quartz, silicon, aluminum and various transition-metal plates with an area of $100 \mathrm{~mm}^{2}$ and a thickness of $50 \mu \mathrm{m}$.

For our experiment on the formation of nickel silicide, nickel films with a thickness of approximately60 nm were deposited on silicon wafers with an area of $100 \mathrm{~mm}^{2}$ and a thickness of $400 \mu \mathrm{m}$ by electron beam evaporation. We studied the formation of nickel silicide by a scanning transmission electron microscope (FEI Company Tecnai Osiris) with an energy dispersive x-ray analysis system (STEM-EDX).

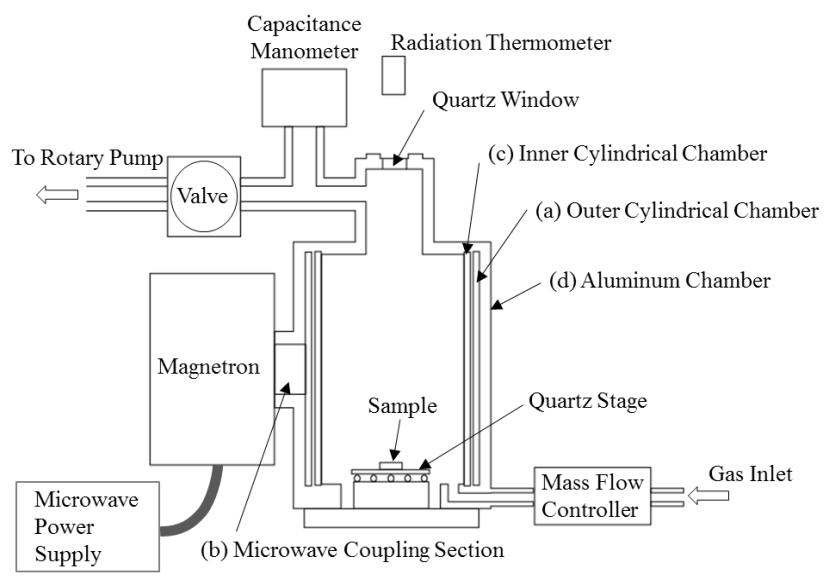

Figure 1. Schematic illustration of apparatus. The inner chamber has a diameter of $131 \mathrm{~mm}$ and a height of $206 \mathrm{~mm}$. 


\section{Results and Discussion}

\subsection{Dependence of Maximum Temperature Dependences on Transition Metal Species}

Figure 2 shows a typical example of the temperature profile obtained using a nickel plate under hydrogen microwave plasma exposure. The temperature rapidly increased to above $900^{\circ} \mathrm{C}$ within $5 \mathrm{~s}$ of turning on the microwave power. When the microwave power was turned off, the temperature suddenly decreased to less than $500^{\circ} \mathrm{C}$ within $5 \mathrm{~s}$. By using Equation (1), the density of hydrogen atoms was determined to be $3.1 \times 10^{21} \mathrm{~m}^{-3}$.

Figure 3 shows the maximum temperatures obtained using various transition-metal species. The maximum temperatures were higher than $800^{\circ} \mathrm{C}$. In contrast, for other materials such as quartz, silicon, and aluminum, we could not observe the heat phenomenon using the radiation thermometer, even when the materials were exposed to the hydrogen plasma for longer than 60 seconds. These results indicate that we can selectively heat only the areas of transition metals deposited on silicon wafers.

\subsection{Nickel Silicide Formation}

Figure 4 shows temperature profiles when nickel film deposited on silicon wafer was exposed to hydrogen microwave plasma, and hydrogen microwave plasma was turned on and off without any sample. In the case of the nickel films, the temperature increased to approximately $450^{\circ} \mathrm{C}$ within $5 \mathrm{~s}$ of turning on the microwave power and suddenly decreased to approximately $380^{\circ} \mathrm{C}$. This result indicates that the amount of surface nickel atoms decreased due to nickel slicidation reaction. When the microwave power was turned off, the temperature suddenly dropped down to the lowest measureable value (approximately $280^{\circ} \mathrm{C}$ ). Since the radiation thermometer

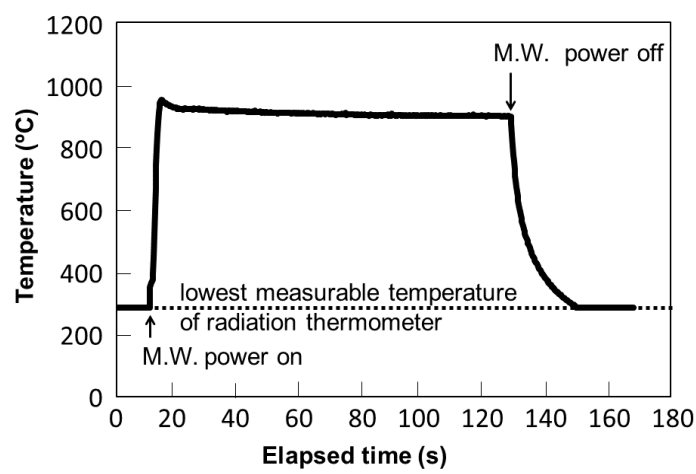

Figure 2. Temperature profile obtained using nickel plate exposed to hydrogen microwave plasma at input microwave power of $1000 \mathrm{~W}$, hydrogen gas flow rate of $10 \mathrm{sccm}$, and pressure of $30 \mathrm{~Pa}$.

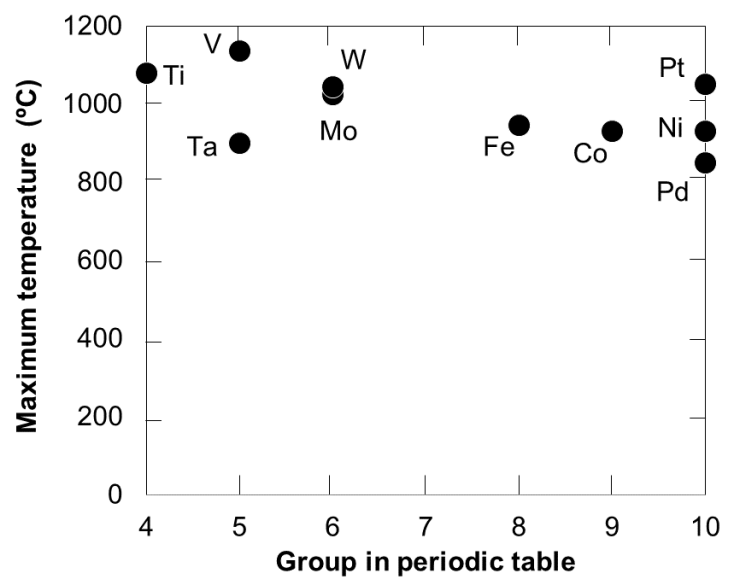

Figure 3. Obtained maximum temperatures when various transition-metal plates were exposed to hydrogen microwave plasma. 
measures the intensity of light from hydrogen plasma, the temperature drop caused by turning off the microwave power is due to the emission of light from hydrogen plasma.

Figure 5 shows a cross-sectional STEM image of the sample after hydrogen plasma exposure for $35 \mathrm{~s}$. It is clearly seen that a nickel silicide layer is formed on the silicon wafer.

Figure 6 shows an EDX line scan profile of the sample in Figure 5, in which the signal intensity is calibrated. The atomic ratio of silicon to nickel is approximately 1:1.

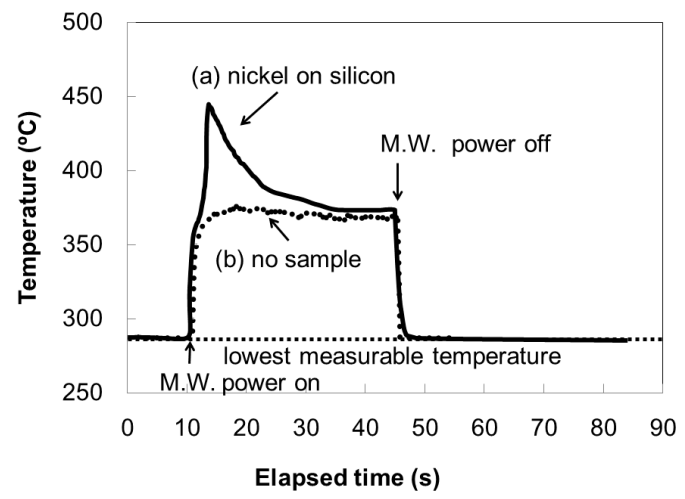

Figure 4. (a) Temperature profile when nickel film deposited on silicon wafer was exposed to hydrogen microwave plasma, and (b) apparent temperature profile when hydrogen microwave plasma was turned on and off without any sample since the radiation thermometer measured the intensity of light from hydrogen plasma.

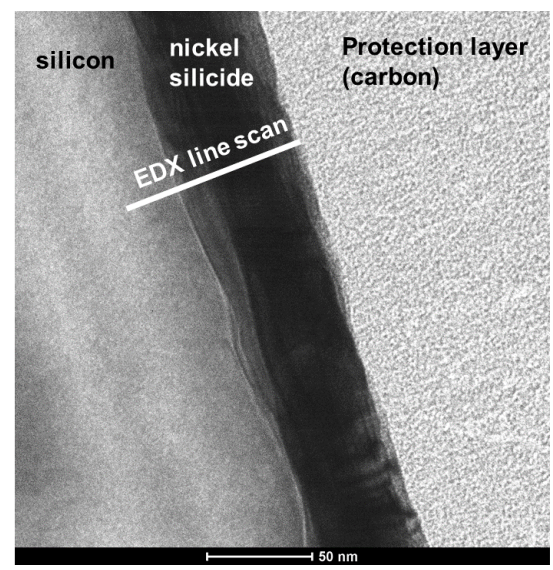

Figure 5. Cross-sectional STEM image of sample in Figure 4.

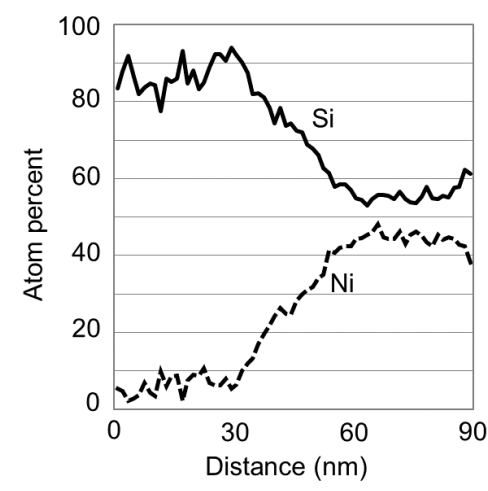

Figure 6. EDX line scan profile of sample in Figure 5. The solid line denotes the silicon atomic percent in the sample and the dashed line the nickel atomic percent. 
Therefore, the dark layer in Figure 5 is made of nickel monosilicide.

From these results, it is assumed that nickel and silicon alloying starts and this reaction continues untill the entire surface nickel layer forms an alloy with silicon. As a result, the heat phenomenon is markedly weakened and the temperature decreases to approximately $380^{\circ} \mathrm{C}$ automatically. In addition, when the samples after nickel silicidation are exposed to hydrogen plasma again, the temperature does not exceed the temperature corresponding to the intensity of light from the hydrogen plasma (approximately $380^{\circ} \mathrm{C}$ ). These results indicate that the high-resistivity silicon rich phase of nickel disilicide is not formed by this heating method. Tinani and

coworkers [12] reported that the formation of nickel disilicide requires a high temperature of above $750^{\circ} \mathrm{C}$.

From Figure 4, it can be seen that the temperature of the sample does not increase to above $450^{\circ} \mathrm{C}$. Thus, nickel disilicide is not formed by this heating method. From the standpoint of Si-ULSI manufacturing, this heating method has advantages: it can selectively heat only the areas of transition metals deposited on silicon wafers, it has a self-stopping mechanism, and it does not cause any heat damage to the other areas. Therefore, this method can improve the reliability of Si-ULSI and is suitable for forming nickel silicide electrodes in silicon wafers with large diameters.

\section{Conclusions}

In this study, we have developed an apparatus with a self-tuning microwave plasma system to form high-density hydrogen plasma and we have confirmed that transition metals can be heated at approximately $1000^{\circ} \mathrm{C}$ by exposure to high-density hydrogen plasma. We achieved the selective heating of nickel layers deposited on silicon wafers and successfully formed nickel silicide. We showed that this phenomenon stopped automatically after silicidation finished. This method is considered to be applicable to the formation of slicides such as tungsten silicide and platinum silicide.

\section{Acknowledgements}

This work was partially supported by Grants-in-Aid for Scientific Research (No. 25390065).

\section{References}

[1] Iwai, H. and Ohmi, S. (2002) Silicon Integrated Circuit Technology from Past to Future. Microelectronics Reliability, 42, 465-491. http://dx.doi.org/10.1016/S0026-2714(02)00032-X

[2] Baeri, P., Grimaldi, M.G., Rimini, E. and Celotti, G. (1983) Pulsed Laser Irradiation of Nickel Thin Films on Silicon. Journal de Physique Colloques, 44, 449-454. http://dx.doi.org/10.1051/jphyscol:1983566

[3] D’Anna, E., Leggieri, G. and Luches, A. (1998) Laser Synthesis of Metal Silicides. Applied Physics A, 45, 325-335. http://dx.doi.org/10.1007/BF00617939

[4] Bohac, V., D’Anna, E., Leggieri, G., Luby, S., Luches, A., Majkova, E. and Martino, M. (1993) Tungsten Silicide Formation by XeClExcimer-Laser Irradiation of W/Si Samples. Applied Physics A, 56, 391-396. http://dx.doi.org/10.1007/BF00324361

[5] Mozetic, M., Drobnic, M., Pregelj, A. and Zupan, K. (1996) Determination of Density of Hydrogen Atoms in the Ground State. Vacuum, 47, 943-945. http://dx.doi.org/10.1016/0042-207X(96)00098-X

[6] Ricard, A., Gaillard, M., Monna, V., Vesel, A. and Mozetic, M. (2001) Excited Species in $\mathrm{H}_{2}, \mathrm{~N}_{2}, \mathrm{O}_{2} \mathrm{Microwave}$ Flowing Discharges and Post-Discharges. Surf. Coat. Technol., 142-144, 333-336. http://dx.doi.org/10.1016/S0257-8972(01)01276-2

[7] Mozetic, M., Vesel, A., Monna, V. and Ricard, A. (2003) H Density in a Hydrogen Plasma Post-Glow Reactor. Vacuum, 71, 201-205. http://dx.doi.org/10.1016/S0042-207X(02)00737-6

[8] Mozetic, M. and Vesel, A. (2005) Density of Neutral Hydrogen Atoms in a Microwave Hydrogen Plasma Reactor. Proceedings of the International Conference Nuclear Energy for New Europe 2005, Bled, 5-8 September 2005, 147.1147.6.

[9] Cvelbara, U., Mozetic, M., Poberaj, I., Babic, D. and Ricard, A. (2005) Characterization of Hydrogen Plasma with a Fiber Optics Catalytic Probe. Thin Solid Films, 475, 12-16. http://dx.doi.org/10.1016/j.tsf.2004.08.047

[10] Vesel, A., Drenik, A., Mozetic, M. and Balat-Pichelin, M. (2010) Hydrogen Atom Density in a Solar Plasma Reactor. Vacuum, 84, 969-974. http://dx.doi.org/10.1016/j.vacuum.2010.01.032

[11] Takamatsu, T. (2003) High Frequency Reaction Processing System. The International Publication No. WO $03 / 096769$.

[12] Tinani, M., Mueller, A., Gao, Y., Irene, E.A., Hu, Y.Z. and Tay, S.P. (2001) In Situ Real-Time Studies of Nickel Silicide Phase Formation. Journal of Vacuum Science \& Technology B, 192, 376-383. http://dx.doi.org/10.1116/1.1347046 